\title{
Spectrophotometric evaluation of 5-layer acrylic teeth hyperpigmentation caused by selected food colors: In vitro study
}

\section{Spektrofotometryczna ocena przebarwień wywołanych wybranymi barwnikami spożywczymi na 5-warstwowych zębach akrylowych - badanie in vitro}

\author{
Magdalena Barzyk1,A-C, Joanna Smardz 2,C,D,F, Włodzimierz Więckiewicz ${ }^{1, A, E, F}$ \\ ${ }^{1}$ Department of Dental Prosthetics, Faculty of Dentistry, Wroclaw Medical University, Poland \\ ${ }^{2}$ Department of Experimental Dentistry, Faculty of Dentistry, Wroclaw Medical University, Poland \\ A - research concept and design; $\mathrm{B}$ - collection and/or assembly of data; $\mathrm{C}$ - data analysis and interpretation; \\ $D$ - writing the article; $E$ - critical revision of the article; $F$ - final approval of the article
}

Address for correspondence

Magdalena Barzyk

E-mail: magdalena_barzyk@tlen.pl

\section{Funding sources}

None declared

\section{Conflict of interest}

None declared

Received on December 15, 2017

Reviewed on February 1, 2018

Accepted on March 21, 2018

DOI

$10.17219 / \mathrm{dmp} / 87116$

Copyright

○ 2018 by Wroclaw Medical University

and Polish Dental Society

This is an article distributed under the terms of the

Creative Commons Attribution Non-Commercial License

(http://creativecommons.org/licenses/by-nc-nd/4.0/)

\begin{abstract}
Background. Prosthetic treatment restores proper chewing function and facial aesthetics, and enhances the quality of life. The overall aesthetic result of the use of removable prostheses is correlated with the type of artificial teeth used and with the maintenance of aesthetic effect and color stability.

Objectives. The aim of the study was to evaluate the formation and intensity of discoloration caused by the dyes present in selected 5 -layer acrylic teeth.
\end{abstract}

Material and methods. The study material was a group of 905 -layer acrylic teeth. Samples were immersed in black tea and dry red wine for 6 months. The control group was immersed in distilled water and consisted of 30 samples. The teeth were subjected to spectrophotometric examination at the start of the study, after $24 \mathrm{~h}, 7$ days, 14 days, 1 month, 4 months, and 6 months.

Results. Among the teeth examined, in about 85\% of the samples the color corresponded with the order. Teeth immersed in black tea became darker. From the $7^{\text {th }}$ day to the $6^{\text {th }}$ month, none of the samples changed their brightness. The teeth had the same degree of brightness. The teeth did not change tone during the experiment. Teeth immersed in dry red wine changed their shade to $\mathrm{A}$ starting on the $14^{\text {th }}$ day, and by the $4^{\text {th }}$ month all samples had changed shade to $\mathrm{A}$ or $\mathrm{C}$. The 5 -layer teeth had quite high shade stability until the $14^{\text {th }}$ day of observation. In the control group, the teeth did not change tone and individual brightness changed more in the direction of brighter shades and less in the darker direction. In the study group, 93\% of the samples did not change their brightness.

Conclusions. Both black tea and dry red wine cause tooth discoloration, more intensely in the case of the

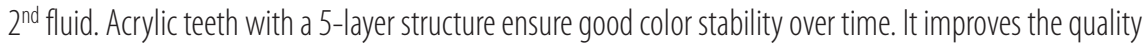
and aesthetics of the restorations used.

Key words: spectrophotometry, acrylic resins, artificial teeth

Słowa kluczowe: spektrofotometria, tworzywa akrylowe, sztuczne zęby 
Prosthetic treatment restores the proper function of the masticatory system and facial aesthetics, and also affects the improvement of the quality of life. The main aim of the dentist is to combine science and art to achieve the optimal functionality and aesthetic effect, which allows the natural look and feel of attractiveness to be restored. Four basic determinants affect the aesthetics of the prosthetic restoration: position, contour, texture, and color of the teeth. Although color is not an important factor affecting denture function, from the physiological point of view, it can have a decisive influence on the process of acceptance. Aesthetics in clinical dentistry offers almost unlimited and exciting challenges for practitioners. In our society, it is believed that appearance significantly affects professional and personal success. It is increasingly expected from the dentists to improve also the smile and appearance of the patient. Knowledge of the concept of color and understanding the nature of light, perception and interpretation of the image is crucial to achieve therapeutic success. Of the many different elements that influence the perception of image, color is considered the most important factor. ${ }^{1-13}$

The overall aesthetic result in treatment with the use of removable dentures is significantly correlated with the type of artificial teeth used and their color stability. Color stability, i.e., retaining color in a specific environment, is an important property of many materials used in dentistry. The use of artificial teeth made of acrylic resin is common due to their beneficial properties, good binding to the prosthesis, low weight, and low fracture rate. Conventional acrylic teeth also have a number of disadvantages, such as poor aesthetic effect, higher wear and susceptibility to discoloration. In order to overcome these problems, manufacturers have introduced numerous modifications that result in the improvement of the quality of acrylic teeth. ${ }^{14,15}$

Discoloration of acrylic resins can be caused by many factors. Internal factors, such as the degree of conversion and amount of residual monomer, can affect the color stability. Another possible source of color change is porosity. The color stability of acrylic teeth is associated with eating habits. It has been reported that some beverages, such as coffee, tea and wine, and the effects of cleaning agents, tobacco, saliva composition, and hygiene habits, can cause discoloration. Changing the color of the resin can lead to aesthetic problems. In order to minimize the color change, it is necessary to choose the right materials from which the artificial teeth are made. ${ }^{15,16}$

The aim of the study was to assess the formation and intensity of discolorations of acrylic 5-layer teeth induced by the dyes present in selected food products.

\section{Material and methods}

The research material consisted of a group of 90 readymade 5-layer acrylic teeth (medial upper incisors) in B3 color (according to information provided by the manufac- turer) (Ivoclar Vivadent AG, Liechtenstein). The research material was divided into 3 equal groups:

- group 1 - samples immersed in distilled water as a control group (30 samples),

- group 2 - samples immersed in black tea (30 samples),

- group 3 - samples immersed in dry red wine (30 samples).

Each specimen was marked individually by engraving a number from 1 to 30 in each test group on the side of the acrylic tooth, which allowed fully repeatable measurements.

The teeth from the $1^{\text {st }}$ group, constituting the control group, were immersed in distilled water. Teeth from the $2^{\text {nd }}$ group were immersed in black tea. The tea solution was obtained by immersing 5 pre-packaged black tea bags weighing 2 g each in $1000 \mathrm{~mL}$ of boiling distilled water for $10 \mathrm{~min}$. After cooling to $37^{\circ} \mathrm{C}$, the experimental solution was filtered through a filter paper. ${ }^{17}$ The $3^{\text {rd }}$ group of samples was immersed in dry red wine.

The material was immersed for 6 months in the fluids in closedcontainersandstoredatroom temperature $\left(22-24^{\circ} \mathrm{C}\right)$ in the absence of light.

The spectrophotometer SpectroShade Micro ${ }^{\circledR}$ (MHT Optic Research, Niederhasli, Switzerland) was used for the objective assessment of the degree of discoloration, which allowed analysis with reference to a standard determined according to the Vita colorant VITAPAN ${ }^{\circledR}$ Classical (Vita Zahnfabrik, Bad Säckingen, Germany).

The spectrophotometer calibration was 2-stage and included the positioning of the handle for white and green tiles. The system analyzed 3 basic components of color: brightness, saturation and color. The following aspects were also analyzed: transparency and transluminescence, base color and component color. A full color map of each tooth was also performed. Using a special scanner (SpectroShade Micro; MHT Optic Research, Niederhasli, Switzerland), a picture of the selected tooth was obtained. The device made it possible to obtain reproducible results by placing the analyzed tooth in a specific position on the device display. The measurement of each sample at the time of testing was performed 3 times on a matte black background, using intraoral attachments provided by the manufacturer. This procedure made it possible to create conditions similar to those in the clinical situation.

The individual color determinations were assigned numbers from 1 to 16 , according to the decreasing brightness determined using the colorant. In total, 7 measurements were made: at the beginning of the study, after $24 \mathrm{~h}$, after 7 days, after 14 days, after 1 month, after 4 months, and after 6 months. The analysis of the results was performed using STATISTICA PL software v. 12 (StatSoft, Tulsa, USA). Descriptive statistics and non-parametric tests, i.e., Pearson's $\chi^{2}$ test and $\chi^{2} \mathrm{NW}$ were used to analyze the qualitative data. For quantitative data analysis, parametric t-tests and a Tukey's honest significant difference test were conducted. Normal distribution of variables was tested using Shapiro-Wilk test and Levene's test. The significance level was set at $\mathrm{p}=0.05$. 


\section{Results}

\section{Change in the color of the teeth subjected to the influence of particular liquids}

\section{Color change of teeth in distilled water}

At the beginning of the experiment, the control group of 5-layer teeth was found to be in the B3 color given by the manufacturer in $94.44 \%$ of cases. A statistically significant change in color occurred after 14 days $(\mathrm{p}=0.044869)$ and after 1 month. In the remaining stages of the experiment, the color changed to a slightly lighter one, but these changes were not statistically significant. At all stages, the 5-layered teeth were in the B3 (87.78-98.89\%) and B4 (1.11-12.22\%) colors. None of the samples changed the color tone. The change of sample brightness in distilled water during the experiment is shown in Table 1.

Table 1. Number of samples that changed brightness in distilled water during the experiment

\begin{tabular}{|l|c|c|c|}
\hline \multicolumn{1}{|c|}{ Time } & No change & Brighter & Darker \\
\hline $24 \mathrm{~h}$ & 88 & 2 & 0 \\
7 days & 85 & 4 & 1 \\
14 days & 86 & 4 & 0 \\
1 month & 86 & 4 & 0 \\
4 months & 84 & 4 & 2 \\
6 months & 84 & 4 & 2 \\
\hline
\end{tabular}

\section{Color change of teeth in black tea}

At the beginning of the experiment, in the group of teeth immersed in black tea, the B3 color given by the manufacturer was noted in $84.44 \%$ of cases. The rest of the group was in the B4 color (15.56\%). After $24 \mathrm{~h}$, none of the samples changed color. After 7 days of the experiment, all samples reached the B4 color and did not change it later. The color from the initial period point compared to the other stages differed significantly $(\mathrm{p}=0.0000)$. The change in sample brightness in black tea during the experiment is shown in Table 2 .

Table 2. Number of samples that changed brightness in black tea during the experiment

\begin{tabular}{|l|c|c|c|}
\hline \multicolumn{1}{|c|}{ Time } & No change & Brighter & Darker \\
\hline $24 \mathrm{~h}$ & 90 & 0 & 0 \\
7 days & 14 & 0 & 76 \\
14 days & 14 & 0 & 76 \\
1 month & 14 & 0 & 76 \\
4 months & 14 & 0 & 76 \\
6 months & 14 & 0 & 76 \\
\hline
\end{tabular}

\section{Color change of teeth in dry red wine}

At the beginning of the experiment, the $\mathrm{B} 3$ color given by the manufacturer was reported in $76.56 \%$ of cases. The rest of the group was in the B4 color (24.44\%). After 24 $\mathrm{h}$, the percentage of samples in the B3 color dropped significantly $(62.22 \%)(\mathrm{p}<0.05)$. After 7 days, the group entirely turned into the B4 color. After 14 days, the percentage of the B4 color (86.87\%) decreased, but new colors, A3.5 and A4, appeared (each color 6.67\%). After 1 month, the percentage of samples with the B4 color decreased (47.78\%), while the A3.5 (22.22\%) and A4 (30\%) colors increased their share. After 4 months, the B tone disappeared and there were only 2 colors, A4 (88.89\%) and C4 (11.11\%). After 6 months, there were still only 2 colors, A4 (74.44\%) and C4 (25.56\%). At each of the examined stages, statistically significant changes occurred compared to the previous stages $(\mathrm{p}<0.05)$. The change in brightness of samples immersed in dry red wine during the experiment is shown in Table 3. The percentage distribution of shades in the test group immersed in dry red wine over the duration of the experiment is shown in Fig. 1.

Table 3. Number of samples that changed brightness in dry red wine during the experiment

\begin{tabular}{|l|c|c|c|}
\hline \multicolumn{1}{|c|}{ Time } & No change & Brighter & Darker \\
\hline $24 \mathrm{~h}$ & 78 & 0 & 12 \\
7 days & 22 & 0 & 68 \\
14 days & 22 & 0 & 68 \\
1 month & 20 & 0 & 70 \\
4 months & 0 & 0 & 90 \\
6 months & 0 & 0 & 90 \\
\hline
\end{tabular}

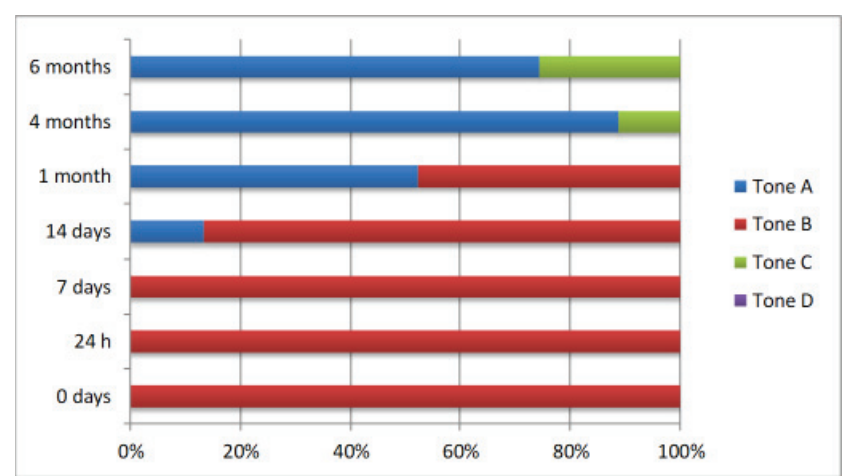

Fig. 1. Percentage distribution of shades in the group immersed in dry red wine during the experiment

\section{Comparison of the ability of the tested fluids to cause discoloration}

At the beginning of the experiment, the teeth differed statisticallysignificantlyin terms of the degree ofcolor between the distilled water group and the wine group $(\mathrm{p}<0.05)$. 
At the $24 \mathrm{~h}$ stage, the effect of the wine was significantly stronger than the influence of tea or distilled water on teeth color $(\mathrm{p}<0.05)$, and the influence of tea and the effect of distilled water on the color of teeth was similar ( $p>0.05)$. After 7 and 14 days, the influence of distilled water was the smallest $(p<0.05)$. In the remaining stages, the influences of individual factors were significantly different $(\mathrm{p}<0.05)$; the smallest in case of distilled water, the largest in case of dry red wine. The percentage distribution of average values of the degree of brightness for particular solutions during the experiment is presented in Fig. 2.

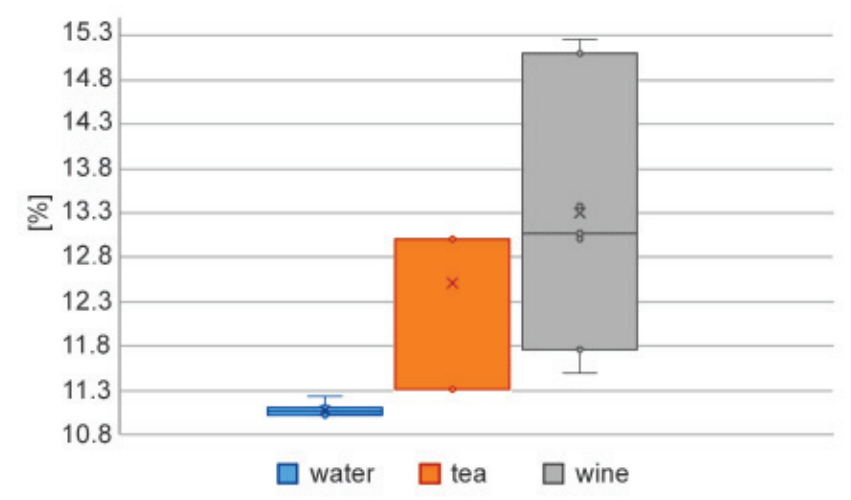

Fig. 2. Average brightness values depending on the solution used

\section{Discussion}

The color and appearance of teeth is a complex phenomenon depending on many factors, such as: transparency, lighting conditions, smoke, light scattering, and gloss. The human eye and brain also affect the overall perception of tooth color. Visual perception can be disturbed by various factors, such as: lighting conditions, gingival color and the closest surroundings, as well as by the type and shape of the used colorant and its position relative to the tooth. ${ }^{1,6,7,12}$ Discoloration of dental materials, including acrylic teeth used in removable dentures, can be assessed visually or by means of spectrophotometric analysis.

Five-layer teeth, according to the manufacturer, are made of 5 layers of different translucency, without the use of additional fillers, thanks to which they give a metameric effect, causing them to be perceived as natural teeth. The use of 5-layer acrylic teeth allows a highly-valued so-called chameleon effect to be obtained. They are characterized by very high durability, wear resistance and biocompatibility. If there is not enough space in the bite, when the dental technician has to make a correction, the 5-layer teeth still retain their color and translucency. These teeth are character- ized by a $40 \%$ lower absorption (absorption of moisture by acrylic), with $56 \%$ less discoloration caused by food and liquids. They have a higher wear resistance compared to the average values of other acrylic teeth.

The study material was a group of 90 5-layer acrylic teeth. The samples were immersed in food liquids, i.e., black tea (30 samples) and dry red wine (30 samples), for 6 months. The control group immersed in distilled water also consisted of 30 samples of 5-layer acrylic teeth.

Among the examined 5-layer teeth, in approx. 85\% of the samples the color of the delivered teeth corresponded with the color of the order. Five-layer teeth immersed in black tea mostly became darker. From the $7^{\text {th }}$ day and up to the $6^{\text {th }}$ month, none of the samples changed their brightness. The teeth had the same degree of brightness and did not change their tone during the experiment. Five-layer acrylic teeth immersed in dry red wine changed shade to A starting on the $14^{\text {th }}$ day, and by the $4^{\text {th }}$ month all samples had changed their shade to A or C. Five-layer teeth showed fairly high shade stability up to the $14^{\text {th }}$ day of observation (Fig. 1). The teeth did not change tone in the control group; in this group, there were individual changes in brightness towards lighter shades and slightly weaker towards darker ones. In the above study group, $93 \%$ of the samples did not change brightness (Table 1 ).

The research shows that both black tea and dry red wine cause the discoloration of acrylic teeth, which is more intense in the case of the $2^{\text {nd }}$ liquid. These results are consistent with other studies performed in vitro. ${ }^{18,19}$ Black tea has the ability to induce yellow discoloration and red wine changes the color of the teeth to a cyanotic shade. ${ }^{18}$ This phenomenon is also confirmed by our own results. Dark brown, easy to remove sediment appeared on the samples immersed in black tea. The combination of dietary chromogens found mainly in tea can cause surface precipitation reactions without the formation of metal sulfides. Both the concentration of the colorant and the exposure time can affect the degree of discoloration of acrylic resin. ${ }^{20-25}$ The surface structure also determines the amount and direction of light reflection. With a smoother surface, the tooth appears brighter. The dyeing of acrylic resins is also dependent on the contact angle and water sorption. The higher the contact angle and lower the water sorption, the lower the tendency to become discolored. These parameters are related to the smoothness of the surface and the porosity. A lower porosity of the surface also reduces the degree of sorption of food dyes. Greater smoothness has a positive effect on increasing the contact angle. ${ }^{20}$

In conclusion, this research confirmed that both black tea and dry red wine cause the discoloration of acrylic teeth, which is more intense in the case of the $2^{\text {nd }}$ liquid. Acrylic teeth with a 5-layer structure provide good color stability over time. This has a positive effect on the quality and aesthetics of the prosthetic restorations used. 


\section{References}

1. Goodacre CJ, Sagel PA. Dental Esthetics in Practice: Part 3 - understanding Color \& Shade Selection. Crest Oral-B at dentalcare.com Continuing Education Course. 2011;27:1-15.

2. Kolek Z. Psychophysical aspects of color [in Polish]. Proceedings of the Electrotechnical Institute. 2010;244:5-15.

3. Sikri VK. Color: Implications in dentistry. J Conserv Dent. 2010;13:249-255.

4. Stopyra W. Compendium of ophthalmology: Seeing colors [in Polish]. Ophthalmol. 2012;3:4-14.

5. Bhat V, Prasad DK, Sood S, Bhat A. Role of colors in prosthodontics: Application of color science in restorative dentistry. Indian J Dent Res. 2011;22:804-809.

6. Shammas M, Alla RK. Color and shade matching in dentistry. Trends Biomater Artif Organs. 2011;25:172-175.

7. Joiner A, Hopkinson I, Deng Y, Westland S. A review of tooth color and whiteness. J Dent. 2008;36(Suppl 1):S2-7.

8. Abhishek A, Prashanti E, Kiran KKS. Digital shade matching: An insight. Res J Pharm Biol Chem Sci. 2015;6:1072-1079.

9. Gómez-Polo C, Gómez-Polo M, Celemin-Viñuela A, Martínez Vázquez De Parga JA. Differences between the human eye and the spectrophotometer in the shade matching of tooth colour. J Dent. 2014;42:742-745.

10. Baharin SA, Tey YD, Tan WJ. Anterior tooth shade selection procedure: Influence of light sources and patient's position. Sains Malays. 2013;42:7-11.

11. Todorović A, Todorović A, Spadijer-Gostović A, Lazić V, Milicić B, Đurišić $S$. Reliability of conventional shade guides in teeth color determination [in Serbian]. Vojnosanitetski Pregled. 2013;70:929-934.

12. Salman A, Habib SR, Azad A. Scientific and artistic principles of tooth shade selection: A review. Pak Oral Dental J. 2011;31:222-226.

13. Yuan JC, Brewer JD, Monaco EA, Davis EL. Defining a natural tooth color space based on a 3-dimensional shade system. J Prosthet Dent. 2007;98:110-119.

14. Koksal T, Dikbas I. Color stability of different denture teeth materials against various staining agents. Dent Mater J. 2008;27:139-144.

15. Madhyastha PS, Kotian R. Effect of staining solutions on color stability of acrylic denture base resins: A spectrophotometric evaluation. Res J Pharm Biol Chem Sci. 2013;4:549-559.

16. Singh SV, Priyanki A. Effect of tea, coffee and turmeric solutions on the color of denture base acrylic resin: An in vitro study. J Indian Prosthodont Soc. 2012;12:149-153.

17. Scotti R, Mascellani SC, Forniti E. The in vitro color stability of acrylic resins for provisional restorations. Int J Prosthodont. 1997;10:164-168.

18. Michalczewski G. Spectrophotometric evaluation of discolorations of dental materials caused by food-origin dyes in an in vitro study [Doctoral thesis] [in Polish]. Lublin, Poland: Medical University of Lublin; 2013.

19. Fujita M, Kawakami S, Noda M, Sano H. Color change of newly developed esthetic restorative material immersed in food-simulating solutions. Dent Mater J. 2006;25:352-359.

20. Kohli S, Bhatia S. Evaluation of the color durability of acrylic resin veneer materials after immersion in common beverages at different time intervals: A spectrophotometric study. Biomed J. 2015;38:244-249.

21. Sepúlveda-Navarro WF, Arana-Correa BE, Borges CPF, Jorge JH, Urban VM, Campanha NH. Color stability of resins and nylon as denture base material in beverages. J Prosthodont. 2011;20:632-638.

22. Bayindir F, Kürklü D, Yanikoğlu ND. The effect of staining solutions on the color stability of provisional prosthodontic materials. J Dent. 2012;40(Suppl 2):41-46.

23. Hersek N, Canay S, Uzun G, Yildiz F. Color stability of denture base acrylic resins in three food colorants. J Prosthet Dent. 1999;81:375-379.

24. Kurtulmus-Yilmaz S, Deniz ST. Evaluation of staining susceptibility of resin artificial teeth and stain removal efficacy of denture cleansers. Acta Odontol Scand. 2014;72:811-818.

25. Makhija PP, Shigli K, Awinashe V. Evaluating the efficacy of denture cleansing materials in removal of tea and turmeric stains: An in vitro study. Indian J Dent Res. 2016;27:528-534. 
